\title{
Thanks to reviewers 2018
}

(c) Società Italiana di Ultrasonologia in Medicina e Biologia (SIUMB) 2018

A scientific journal's greatest responsibility is to ensure that all contributions accepted for publication are rigorously but fairly reviewed. We gratefully acknowledge the valued support of the following scientists who have reviewed papers for the Journal of Ultrasound in 2018.

\author{
Agati Luciano, Italy \\ Aliotta Antonio, Italy \\ Ascenti Giorgio, Italy \\ Aslan Ahmet, Turkey \\ Badea Radu, Romania \\ Balconi Giuseppe, Italy \\ Bartolotta Tommaso Vincenzo, Italy \\ Batticciotto Alberto, Italy \\ Belli Paolo, Italy \\ Bertolotto Michele, Italy \\ Berzigotti Annalisa, Switzerland \\ Bini Fabiano, Italy \\ Boccatonda Andrea, Italy \\ Bolondi Luigi, Italy \\ Bortolotto Chandra, Italy \\ Brkljacic Boris, Croatia \\ Caiazzo Corrado, Italy \\ Calliada Fabrizio, Italy \\ Cantisani Vito, Italy \\ Capone Fabrizio, Italy \\ Cardobi Nicolo, Italy \\ Caremani Marcello, Italy \\ Carriero Alessandro, Italy \\ Casadei Alder, Italy \\ Castellani Sergio, Italy \\ Catalano Orlando, Italy \\ Catalini Roberto, Italy \\ Cavalli Roberta, Italy \\ Cavanna Luigi, Italy \\ Čavka Mislav, Croatia \\ Coley Brian, United States Minor Out- \\ lying Islands \\ Corcioni Beniamino, Italy \\ David Emanuele, Italy \\ De Sio Ilario, Italy \\ Del Medico Marta, Italy \\ Delic-Brkljacic Diana, Croatia
}

Derchi Lorenzo, Italy

Di Cesare Ernesto, Italy

Di Paola Valerio, Italy

Di Serafino Marco, Italy

Dietrich Christoph F., Germany

Draghi Ferdinando, Italy

Drudi Francesco M., Italy

Esposito Francesco, Italy

Ewertsen Caroline, Denmark

Faverzani Stefano, Italy

Filice Carlo, Italy

Fiorino Fabio, Italy

Francica Giampiero, Italy

Fruganti Alessandro, Italy

Gallet Jacqueline, United States

Garberoglio Roberto, Italy

Gnudi Giacomo, Italy

Granata Antonio, Italy

Grgurevic Ivica, Croatia

Huang Pintong, China

Iezzi Roberto, Italy

Indraccolo Ugo, Italy

Kabaalioglu Adnan, Turkey

Klauser Andrea, Austria

Leone Francesco, Italy

Liberati Marco, Italy

Licata Anna, Italy

Maconi Giovanni, Italy

Manganaro Lucia, Italy

Manola Sime, Croatia

Masciocchi Carlo, Italy

Matijević Ratko, Croatia

Mauri Giovanni, Italy

Miele Vittorio, Italy

Mirk Paoletta, Italy

Moller Ingrid, Spain

Orlandi Davide, Italy
Pavlica Pietro, Italy

Perrone Tiziano, Italy

Poggiani Carlo, Italy

Pompili Maurizio, Italy

Radzina Maija, Latvia

Ramadori Giuliano, Germany

Rapaccini Gian Ludo, Italy

Rea Gaetano, Italy

Roić Goran, Croatia

Romano Marcello, Italy

Rossi Eugenio, Italy

Russo Marco, Italy

Sacerdoti David, Italy

Santoliquido Angelo, Italy

Schiavone Cosima, Italy

Sconfienza Luca, Italy

Serra Carla, Italy

Sidhu Paul, United Kingdom

Sofia Soccorsa, Italy

Soresi Maurizio, Italy

Speca Stefania, Italy

Sperandeo Marco, Italy

Sporea Ioan, Romania

Stramare Roberto, Italy

Testa Antonia Carla, Italy

Thompson Peter, Australia

Tiboni Gian Mario, Italy

Tomà Paolo, Italy

Traikova Nikoleta, Bulgaria

Trombetta Carlo, Italy

Valente Iacopo, Italy

Valentino Massimo, Italy

Vallone Gianfranco, Italy

Vecchiarino Luca, Italy

Vidili Gianpaolo, Italy

Vignoli Massimo, Italy

Zappia Marcello, Italy 